\title{
Quadratic Optimal Control of Fractional Stochastic Differential Equation with Application
}

\author{
Sameer Qasim Hasan, Gaeth Ali Salum \\ College of Education, Almustansryah University, Baghdad, Iraq
}

Email address:

dr.sameer@yahoo.com (S. Q. Hasan),gaithali1993@yahoo.com (G. A. Salum)

\section{To cite this article:}

Sameer Qasim Hasan, Gaeth Ali Salum. Quadratic Optimal Control of Fractional Stochastic Differential Equation with Application. Science Journal of Applied Mathematics and Statistics. Vol. 4, No. 4, 2016, pp. 147-158. doi: 10.11648/j.sjams.20160404.15

Received: May 4, 2016; Accepted: June 3, 2016; Published: July 23, 2016

\begin{abstract}
The paper is devoted to the study of optimal control of Quadratic Optimal Control of Fractional stochastic differential Equation with application of Economy Mode with different types of fractional stochastic formula (ITO, Stratonovich), By using the Dynkin formula, Hamilton-Jacobi-Bellman (HJB) equation and the inverse HJB equation are derived. Application is given to a stochastic model in economics.
\end{abstract}

Keywords: Fractional Stochastic Differential Equations, Dynkine Formula, Hamilton-Jacobi-Bellman Equation

\section{Introduction}

In the following controlled Fractional stochastic differential equations was introduced

1. $x(t)=x(0)+\int_{0}^{t}(H(t) x(t)+M(t) u(t)) d t+\int_{0}^{t} b(t) d B^{H}(t)$.

2. $x(t)=x(0)+\int_{0}^{t}(H(t) x(t)+M(t) u(t)-$

$$
\left.\frac{1}{2} b(t) \frac{d b(t)}{d x}\right) d t+\int_{0}^{t} b(t) d B(t) \text {. }
$$

3. $\mathrm{x}(\mathrm{t})=\mathrm{x}(0)+\int_{0}^{\mathrm{t}}(\mathrm{H}(\mathrm{t}) \mathrm{x}(\mathrm{t})+\mathrm{M}(\mathrm{t}) \mathrm{u}(\mathrm{t})-$ $\left.\frac{1}{2} b(t) \frac{d b(t)}{d x}\right) d t+\int_{0}^{t} b(t) d B^{H}(t)$.

where $x(t), t \in[0, T]$, is a given continuous process, $u(t)$ is a control process, $\mathrm{H}(\mathrm{t})$ be $\mathrm{n} \times \mathrm{n}$ matrices, $M(\mathrm{t})$ be $\mathrm{n} \times \mathrm{k}$ matrices, $b(t)$ be $n \times m$ matrices, the control $u(t)$ be $k \times$ 1 vector, $\mathrm{B}^{\mathrm{H}}(\mathrm{t})$ and $\mathrm{B}(\mathrm{t})$ are Fractional Brownian Motion and Brownian Motion respectively.

we presented Dynkin formula, This result can be obtained from Taylor formula for above Fractional stochastic differential equations and there generators, By using Dynkin formula and the property of expectation, the HamiltonJacobi-Bellman (HJB) equation and the inverse HJB equation have been stated. The stochastic optimal control for the stochastic differential delay equation was found in the paper [1], we will give the proof for Dynkin formula, the HamiltonJacobi-Belman (HJB) equation, the inverse HJB equation and the optimal control for each of the above equation. For a definitions related to optimal control see [2], a Ramsey model $[4,6]$ that takes into account the randomness in the production cycle.

The models is described by the equations

1. $d k(t)=[H(t) k(t)+u(k(t)) M(t)] d t+b(k(t)) d B^{H}$

2. $d k(t)=[H(t) k(t)+M(t) u(k(t))] d t+b(k(t)) \circ d B(t)$

3. $d k(t)=[H(t) k(t)+M(t) u(k(t))] d t+b(k(t)) \circ d B^{H}$ (t)

where $\mathrm{k}$ is the capital, $\mathrm{M}$ is the production, $\mathrm{u}$ is control process, $H(t)$ be $n \times n$ positive matrices. For these stochastic economic models the optimal control for the first and second economic equation is found to be $u(t)=-\frac{\operatorname{Rx}(T) M(t)}{G(t)}$, and the optimal control for the third equation is found to be $\mathrm{u}(\mathrm{t})=-\frac{\mathrm{M}(\mathrm{t}) \mathrm{Rx}(\mathrm{T})}{\mathrm{G}(\mathrm{t})}$, and the optimal performance is

\section{Definitions and Basic Concept}

\section{Definition (1), [3]}

A random experiment is a process that has random out comes.

Definition (2), [3]

A sample space is the set of all possible outcomes of a random experiment and is denoted by $\Omega$.

Definition (3), [3]

A $\sigma$-algebra Fof subset of a sample space $\Omega$ (which is the set of all possible outcomes) satisfies the following

i. $\Omega \in \mathrm{F}$.

ii. If $A \in F$, then $A^{c}$ where $A^{c}$ is the complement of all set A. 
iii. For any sequence $\left\{A_{n}\right\} \subseteq F$ Then $\cup_{n=1}^{\infty} A_{n} \in F$ and $\bigcap_{n=1}^{\infty} A_{n} \in \mathrm{F}$ the element of Fare called measurable sets and the $\operatorname{pair}(\Omega, F)$ is called a measurable space.

Definition(4), [3]

The probability $\mathrm{p}$ is a set function that $\mathrm{p}: \mathrm{F} \rightarrow[0,1]$, and $\mathrm{p}$ is called a probability measure if the following conditions hold

i. $P(\Omega)=1$.

ii. $P\left(A^{c}\right)=1-p(A)$.

iii. $P\left(\bigcup_{i=1}^{n} A_{i}\right)=\sum_{i=1}^{n} p\left(A_{i}\right)$, if $A_{i} \cap A_{j}=\varnothing$, for $i \neq j$.

Definition (5), [3]

The triplet $(\Omega, \mathrm{F}, \mathrm{p})$ consisting of the sample space $\Omega$, the $\sigma$-algebra $\mathrm{F}$ of subset of $\Omega$ and a probability measure $p$ defined on $\mathrm{F}$ is called a probability space.

Definition (6), [3]

A random variable $x$, in the probability space $(\Omega, F, p)$ is a function $\mathrm{x}: \Omega \rightarrow \mathrm{R}$ such that the inverse $\mathrm{x}^{-1}(\mathrm{~A})=\{\mathrm{w} \in \Omega: \mathrm{x}$ (w) $\in \mathrm{A}\} \in \mathrm{F}$, for all open subset A of $\mathrm{R}$.

Definition (7), [3]

A stochastic process $\mathrm{x}:[0, \mathrm{~T}] \times \Omega \rightarrow \mathrm{R}$, in probability space $(\Omega, F, p)$ is a function such that $x(t,$.$) is a random$ variable in $(\Omega, F, p)$ for all $t \in(0, T)$ we will often write $x(t)$ $\equiv \mathrm{x}(\mathrm{t},$.$) .$

Definition (8), [3]

A stochastic process $x=\{x(t), t \in[0, T]\}$ is said to be Gaussian if for all $n \geq 1$ and allt $t_{1}, t_{2}, \ldots \ldots ., t_{n} \in[0, T],\left(x_{t_{1}}\right.$, $\left.\mathrm{x}_{\mathrm{t}_{2}}, \ldots \ldots \ldots, \mathrm{x}_{\mathrm{t}_{\mathrm{n}}}\right)$ is Gaussian random vector. if the mean ofxequal to zero then xis said to be centered.

Definition (9), [3]

A stochastic process $x(t), t \geq 0$, on a probability space $(\Omega$, $\mathrm{F}, \mathrm{P})$ is adapted to the filtration $\left(F_{t}\right) \geq 0$ if for each $\mathrm{t} \geq 0, \mathrm{x}$ (t) is $F_{t}$ - measurable.

$$
\mathrm{D}_{\mathrm{t}} \mathrm{F}=\sum_{j=1}^{n} \sum_{i=1}^{n_{j}} \frac{\partial f_{j}}{\partial x_{i}}\left(\int_{0}^{1} r_{1 j} \mathrm{~dB}^{\mathrm{H}} \ldots \ldots \ldots \int_{0}^{1} \mathrm{r}_{\mathrm{nj}} \mathrm{dB}^{\mathrm{H}}\right) \eta_{j} \otimes r_{i j}(\mathrm{t})
$$

where $\eta_{j} \in \mathrm{V}, r_{k j} \in L^{2} \phi_{H}\left([0,1], L^{2}(\mathrm{U}, \mathrm{R})\right)$.

Definition (14), [2]

A measurable function $\mathrm{f}: R^{\mathrm{n}} \rightarrow[0, \infty]$ is called supermeanvalued with respect to $\mathrm{x}(\mathrm{t})$ iff $(\mathrm{x}) \geq \mathrm{E}_{\mathrm{x}}\left[\mathrm{f}\left(\mathrm{x}_{\mathrm{T}_{\mathrm{H}}}\right)\right]$ for all stopping time $\mathrm{T}$ and all $\mathrm{x} \in R^{\mathrm{n}}$.

Remark(1), [2]

Let $\mathrm{f}_{1}, \mathrm{f}_{2}, \ldots \ldots \ldots, \mathrm{f}_{\mathrm{k}}$ are bounded Borel function on $R^{\mathrm{n}}$ and $\mathrm{T}$ be a stopping time and $F_{\mathrm{T}}$ is $\sigma$-algebra, then

$$
\mathrm{E}_{\mathrm{x}}\left[\mathrm{f}_{1}\left(\mathrm{x}_{\mathrm{T}+\mathrm{h}_{1}}\right), \mathrm{f}_{2}\left(\mathrm{x}_{\mathrm{T}+\mathrm{h}_{2}}\right), \ldots, \mathrm{f}_{k}\left(\mathrm{x}_{\mathrm{T}+\mathrm{h}_{\mathrm{k}}}\right) \mid F_{\mathrm{T}}\right]=\mathrm{E}_{\mathrm{x}_{\mathrm{T}}}\left[\mathrm{f}_{1}\left(\mathrm{x}_{\mathrm{T}+\mathrm{h}_{1}}\right), \mathrm{f}_{2}\left(\mathrm{x}_{\mathrm{T}+\mathrm{h}_{2}}\right), ., \mathrm{f}_{k}\left(\mathrm{x}_{\mathrm{T}+\mathrm{h}_{\mathrm{k}}}\right)\right]
$$

For all $0 \leq h_{1} \leq h_{2} \leq \ldots \ldots \ldots \leq h_{k}$, let $g$ be the set of all real $\mathrm{M}_{\infty}$-measurable function for $\mathrm{t} \geq 0$, we define the shift operator $\theta_{\mathrm{t}}: \mathrm{g} \rightarrow \mathrm{g}$, if $\mathrm{\eta}=\mathrm{y}_{1}\left(\mathrm{x}_{\mathrm{t}_{1}}\right), \mathrm{y}_{2}\left(\mathrm{x}_{\mathrm{t}_{2}}\right), ., \mathrm{y}_{k}\left(\mathrm{x}_{\mathrm{t}_{\mathrm{k}}}\right)$, where $\mathrm{y}_{i}$ is Borel measurable $\mathrm{t}_{i} \geq 0$ then

$\theta_{\mathrm{t}} \eta=\mathrm{y}_{1}\left(\mathrm{x}_{\mathrm{t}+\mathrm{t}_{1}}\right), \mathrm{y}_{2}\left(\mathrm{x}_{\mathrm{t}+\mathrm{t}_{2}}\right), ., \mathrm{y}_{k}\left(\mathrm{x}_{\mathrm{t}+\mathrm{t}_{\mathrm{k}}}\right)$, then it follows from (3) that

$$
\left.\mathrm{E}_{\mathrm{x}}\left[\theta_{\mathrm{t}} \eta\right) \mid F_{\mathrm{T}}\right]=\mathrm{E}_{\mathrm{x}_{\mathrm{T}}}[\eta]
$$

For any stopping time $\alpha$, the following property be satisfy

$$
\mathrm{E}_{\mathrm{x}}\left[\mathrm{f}\left(\mathrm{x}_{\alpha}\right)\right]=\mathrm{E}_{\mathrm{x}}\left[\mathrm{E}_{\mathrm{x}}\left[\mathrm{f}\left(\mathrm{x}_{\mathrm{T}_{\mathrm{H}}}\right)\right]\right]=\mathrm{E}_{\mathrm{x}}\left[\mathrm{E}_{\mathrm{x}}\left[\theta \alpha \cdot \mathrm{f}\left(\mathrm{x}_{\mathrm{T}_{\mathrm{H}}}\right) \mid F_{\mathrm{T}}\right]\right]=\mathrm{E}_{\mathrm{x}}\left[\theta \alpha \mathrm{f} .\left(\mathrm{x}_{\mathrm{T}_{\mathrm{H}}}\right)\right]=\mathrm{E}_{\mathrm{x}}\left[\mathrm{f}\left(\mathrm{x}_{\mathrm{T}_{\mathrm{H}} \alpha}\right)\right] .
$$

where $\mathrm{T}_{\alpha}=\inf \{\mathrm{t}>\alpha\}$

$$
E_{x}\left[f\left(x_{\alpha}\right)\right] \leq E_{x}\left[f\left(x_{T_{H}}\right)\right]=f(x) .
$$

So $\mathrm{f}$ is supermeanvalued 


\section{Fractional Stochastic Differential Equation}

Let $\mathrm{a}(\mathrm{x}(\mathrm{t})), \mathrm{b}(\mathrm{x}(\mathrm{t}))$ are continuous functionaldefined on the metric space $K$, let the Fractional stochastic process $x(t)$ satisfy the Fractional Stochastic Differential Equation

$$
d x(t)=a(x(t)) d t+b(x(t)) d B^{H}(t)
$$

and $\mathrm{B}^{\mathrm{H}}(\mathrm{t})$ is Brownian motion.

Let $H(t)$ be $n \times n$ matrices, $M(t)$ be $n \times k$ matrices, $b(t)$ be $\mathrm{n} \times \mathrm{m}$ matrices and thecontrol $\mathrm{u}(\mathrm{t})$ be $\mathrm{k} \times 1$ vector and $\mathrm{B}^{\mathrm{H}}(\mathrm{t})$ is Brownian motion let

$$
a(x(t))=H(t) x(t)+M(t) u(t)
$$

and

$$
\begin{gathered}
\mathrm{b}(\mathrm{x}(\mathrm{t}))=\mathrm{b}(\mathrm{t}) \\
\mathrm{f}(\mathrm{x}(\mathrm{t}))=\mathrm{f}(\mathrm{x}(0))+\int_{0}^{\mathrm{t}} \frac{\mathrm{d}}{\mathrm{dx}}(\mathrm{x}(\mathrm{t})) \mathrm{a}(\mathrm{t}) \mathrm{dt}+\int_{0}^{\mathrm{t}} \int_{0}^{\mathrm{t}} \frac{\mathrm{d}^{2}}{\mathrm{~d}^{2} \mathrm{x}}(\mathrm{x}(\mathrm{p})) \int_{0}^{\mathrm{p}}\left(\mathrm{D}_{\mathrm{q}} \mathrm{a}(\mathrm{t})\right) \mathrm{dt} \mathrm{b}(\mathrm{p}) \emptyset_{\mathrm{H}}(\mathrm{p}-\mathrm{q}) \mathrm{dqdp} \\
+\int_{0}^{\mathrm{t}} \int_{0}^{\mathrm{p}} \frac{\mathrm{d}^{2}}{\mathrm{~d}^{2} \mathrm{x}}(\mathrm{x}(\mathrm{p})) \mathrm{b}(\mathrm{q}) \mathrm{b}(\mathrm{p}) \emptyset_{\mathrm{H}}(\mathrm{p}-\mathrm{q})+\int_{0}^{\mathrm{t}} \frac{\mathrm{d}^{2}}{\mathrm{~d}^{2} \mathrm{x}}(\mathrm{x}(\mathrm{t})) \mathrm{b}(\mathrm{t}) \mathrm{dB} \mathrm{B}^{\mathrm{H}}(\mathrm{t}) \\
\quad+\int_{0}^{\mathrm{t}} \int_{0}^{\mathrm{t}} \frac{\mathrm{d}^{2}}{\mathrm{~d}^{2} \mathrm{x}}(\mathrm{x}(\mathrm{P})) \int_{0}^{\mathrm{p}}\left(\mathrm{D}_{\mathrm{q}} \mathrm{b}(\mathrm{t})\right) \mathrm{dB}^{\mathrm{H}}(\mathrm{t}) \mathrm{b}(\mathrm{p}) \emptyset_{\mathrm{H}}(\mathrm{p}-\mathrm{q}) \mathrm{dqdp}
\end{gathered}
$$

by taking the derivative of both saided one can get

$$
\begin{aligned}
d f(x(t))=\frac{d}{d x} & (x(t)) a(t) d t+\int_{0}^{t} \frac{d^{2}}{d^{2} x}(x(p)) \int_{0}^{p} D_{q}(a(t)) d t b(p) \emptyset_{H}(p-q) d q d p d t+\int_{0}^{p} \frac{d^{2} f(x(p))}{d^{2} x} b(q) b(p) \emptyset_{H}(p-q) d q d p d t \\
& +\frac{d^{2} f(x(t))}{d^{2} x} b(t) d B^{H}(t) d t+\int_{0}^{t} \frac{d^{2} f(x(p))}{d^{2} x} \int_{0}^{p}\left(D_{q} b(t)\right) d B^{H}(t) b(p) \emptyset_{H}(p-q) d q d p d t .
\end{aligned}
$$

by applying (7) on (13) to get

$$
d f\left(x(t)=\frac{d f(x(t))}{d x}[H(t) x(t)+M(t) u(t)] d t+\int_{0}^{p} \frac{d^{2} f(x(p))}{d^{2} x} b(q) b(p) \emptyset_{H}(p-q) d q d p d t+\frac{d^{2} f(x(t))}{d^{2} x} b(t) d B^{H}(t) d t .\right.
$$

Definition (15) [5]

The generator $\mathrm{A}^{\mathrm{u}}$ of anFractional Stochastic differential equation (7) defined by

$$
\mathrm{A}^{\mathrm{u}} \mathrm{f}=\frac{\mathrm{E}[\mathrm{df}(\mathrm{x}(\mathrm{t}))]}{\mathrm{dt}}
$$

Remark (3)

by substituting equation (15) in equation (16) eyelid that

$$
A^{u} f=\frac{d f(x(t)}{d x}[H(t) x(t)+M(t) u(t)]+\int_{0}^{p} \frac{\left.d^{2} x(p)\right)}{d^{2} x} b(q) b(p) \emptyset_{H}(p-q) d q d p .
$$

\subsection{Fractional Martingale Problem}

If (7) is an ITO Fractional Stochastic Differential Equation with generator $\mathrm{A}^{\mathrm{u}}$ and $\mathrm{f} \in C^{2}(\mathrm{R})$ then the Fractional Martingale formula is 


$$
\mathrm{f}(\mathrm{x}(\mathrm{t}))=\mathrm{f}(\mathrm{x}(0))+\int_{0}^{t} \mathrm{~A}^{\mathrm{u}} \mathrm{fdt}+\frac{\mathrm{d}^{2} \mathrm{f}(\mathrm{x}(\mathrm{t}))}{\mathrm{d}^{2} \mathrm{x}} \mathrm{b}(\mathrm{t}) d \mathrm{~B}^{\mathrm{H}}(\mathrm{t}) d \mathrm{~d}+\int_{0}^{\mathrm{t}} \frac{\mathrm{d}^{2} \mathrm{f}(\mathrm{x}(\mathrm{p}))}{\mathrm{d}^{2} \mathrm{x}} \int_{0}^{\mathrm{p}}\left(\mathrm{D}_{\mathrm{q}} \mathrm{b}(\mathrm{t})\right) d \mathrm{~B}^{\mathrm{H}}(\mathrm{t}) \mathrm{b}(\mathrm{p}) \emptyset_{\mathrm{H}}(\mathrm{p}-\mathrm{q}) d q d p d t .
$$

\subsection{Dynkine Formula for the Linear Quadratic Regulator Problem}

Let $h \in C^{2}(\mathrm{R}), \mathrm{C}(\mathrm{t})$ be the $\mathrm{n} \times \mathrm{n}$ matrices and $\mathrm{G}(\mathrm{t})$ be the $\mathrm{k} \times \mathrm{k}$ matrices, Note that from equation (11) and equation (17) we obtain the following Fractional Taylor formula for the function $\mathrm{h}(\mathrm{x}(\mathrm{t}))$ where $\mathrm{h}(\mathrm{x}(\mathrm{t}))$ defined as

$$
\begin{gathered}
h(x(t))=x^{T}(t) C(t) x(t)+G(t) \\
h(x(t))=h(x(0))+\int_{0}^{t}\left[\frac{d h(x(t))}{d x}[(H(t) x(t)+M(t) u(t)]\right. \\
\left.+\int_{0}^{p} \frac{d^{2} h(x(p))}{d^{2} x} b(q) b(p) \emptyset_{H}(p-q) d q d p\right] d t+\frac{d^{2} h(x(t))}{d^{2} x} b(t) d B^{H}(t) d t
\end{gathered}
$$

Let $\mathrm{T}$ be a stopping time for the stochastic processx ( $\mathrm{t}$ ) defined in equation (12) such that $\mathrm{E}\left(\int_{0}^{\mathrm{T}} \mathrm{A}^{\mathrm{u}} \mathrm{h}(\mathrm{x}(\mathrm{t})) \mathrm{dt}<\infty\right.$, by taking the expectation of two sides, one can get the following Dynkin formula

$$
\begin{gathered}
E(h(x(T)))=h(x(0))+E\left[\int _ { 0 } ^ { T } \left[\frac{d h(x(t))}{d x}\left[(H(t) x(t)+M(t) u(t)] d t+\int_{0}^{p} \frac{d^{2} h(x(p))}{d^{2} x} b(q) b(p) \emptyset_{H}(p-q) d q d p\right] d t E(h(x(T)))\right.\right. \\
=h(x(0))+E\left[\int_{0}^{T} A^{u} h(x(t)) d t\right]
\end{gathered}
$$

\subsection{The Quadratic Regulator Optimal Problem}

Assume that the cost function of the fractional linear quadratic regulator function is

$$
h(x, u)=E\left(x(T)^{T} R x(T)+\int_{0}^{T}\left(x^{T}(t) C(t) x(t)+u^{T}(t) G(t) u(t)\right) d t\right)[5]
$$

where all of the coefficients $C(t)$ be the $n \times n$ matrics, $G(t)$ be the $k \times k$, the control $u(t)$ be $k \times 1$ vector, we assume that $C(t)$ and Rare symmetric, non negative definite and $G(t)$ is symmetric positive definite and $T$ is the final time of the solution $x(t)$ where $x(t)$ defined in (3. 4) such that $E_{x}|T|<\infty$, the problem is to find the optimal control $u^{*}(t)$ such that

$$
\mathrm{h}\left(\mathrm{x}, \mathrm{u}^{*}(\mathrm{t})\right)=\min \{\mathrm{h}(\mathrm{x}, \mathrm{u})\} \text {. }
$$

\section{Hamilton-Jacobi-Bellman Equation for Quadratic Regulator Problem Consider the Markova Control $u(t)=u(x(t))$}

$$
A^{u} h=\frac{d h(x(t)}{d x}[H(t) x(t)+M(t) u(t)]+\int_{0}^{p} \frac{d^{2} h(x(p))}{d^{2} x} b(q) b(p) \emptyset_{H}(p-q) d q d p .
$$

Theorem (1) "HJB equation "

$$
\text { Define } \mathrm{h}^{*}(\mathrm{x})=\min \{\mathrm{h}(\mathrm{x}, \mathrm{u}): \mathrm{u}=\mathrm{u}(\mathrm{t})-\text { Markov control }\}
$$

Suppose that $\mathrm{h} \in C^{2}(\mathrm{R})$ and the optimal controlu* exists Then

$$
\min \left\{\mathrm{x}^{\mathrm{T}}(\mathrm{t}) \mathrm{C}(\mathrm{t}) \mathrm{x}(\mathrm{t})+\mathrm{u}^{\mathrm{T}}(\mathrm{t}) \mathrm{G}(\mathrm{t}) \mathrm{u}(\mathrm{t})+\mathrm{A}^{\mathrm{u}} \mathrm{h}^{*}(\mathrm{x})\right\}=0
$$

where $\mathrm{G}(\mathrm{t})$ be the $\mathrm{k} \times \mathrm{k}$ metrics, the control $\mathrm{u}(\mathrm{t})$ be $\mathrm{k} \times 1$ vector, and the generator $\mathrm{A}^{\mathrm{u}}$ is given by equation (22) and

$$
\mathrm{h}^{*}(\mathrm{x})=\mathrm{x}(\mathrm{T}){ }^{\mathrm{T}} \mathrm{R} x(\mathrm{~T})
$$

The minim is a chivied when $u^{*}$ is optimal. In other words

$$
x^{T}(t) C(t) x(t)+u^{* T}(t) G(t) u^{*}(t)+A^{u^{*}} h^{*}(x)=0
$$

Proof

Now proceed to prove (7. 4), let $\alpha=\operatorname{Tv}$ be the first exit time of the solution $\mathrm{x}(\mathrm{t})$ by using (2. 5) and (2.6)

$$
E_{x}[h(x(\alpha), u)]=E_{x}\left[E_{x}\left[\int_{0}^{T}\left(x^{T}(t) C(t) x(t)+u^{T}(t) G(t) u(t)\right) d t+x(T){ }^{T} R x(T)\right]\right.
$$




$$
\begin{aligned}
=E_{x}\left[E _ { x } \left[\theta_{\alpha} \int_{0}^{T}\right.\right. & \left.\left(x^{T}(t) C(t) x(t)+u^{T}(t) G(t) u(t)\right) d t+x^{T}(t) R x(T) / F_{\alpha}\right] \\
= & E_{x}\left[\int_{\alpha}^{T}\left(x^{T}(t) C(t) x(t)+u^{T}(t) G(t) u(t)\right) d t\right. \\
& \left.-\int_{0}^{\alpha}\left[x^{T}(t) C(t) x(t)+u^{T}(t) G(t) u(t)\right] d t\right]
\end{aligned}
$$

$E_{x}[h(x, u)]=h(x, u)-E_{x}\left[\int_{0}^{\alpha}\left(x^{T}(t) C(t) x(t)+u^{T}(t) G(t) u(t)\right) d t\right]$, Thus

$$
h(x, u)=E_{x}\left[\int_{0}^{\alpha} x^{T}(t) C(t) x(t)+u^{T}(t) G(t) u(t) d t\right]+E_{x}[h(x, u)](28)
$$

$$
h^{*}(x) \leq h(x, u)=E_{x}\left[\int_{0}^{\alpha} x^{T}(t) C(t) x(t)+u^{T}(t) G(t) u(t) d s\right]+E_{x}[h(x, u)]
$$

by equation (21), we get

$$
\begin{aligned}
& E_{x}[h(x, u)]=h(x)+E_{x} \int_{0}^{\alpha} A^{u^{*}} h^{*}(x) d t \\
& h^{*}(x) \leq h(x, u)=E_{x}\left[\int_{0}^{\alpha} x^{T}(t) C(t) x(t)+u^{T}(t) G(t) u(t) d s\right]+h(x) \\
& +\mathrm{E}_{\mathrm{x}} \int_{0}^{\alpha} \mathrm{A}^{\mathrm{u}^{*}} \mathrm{~h}^{*}(\mathrm{x}) \mathrm{dt} \\
& \text { Or } 0 \leq E_{x}\left[\int_{0}^{\alpha} x^{T}(t) C(t) x(t)+u^{T}(t) G(t) u(t) d s\right]+E_{x} \int_{0}^{\alpha} A^{u^{*}} h^{*}(x) d t \\
& \text { At } \alpha \rightarrow 0 \text {. Thus } 0 \leq \mathrm{E}_{\mathrm{x}}\left\{\mathrm{x}^{\mathrm{T}}(\mathrm{t}) \mathrm{C}(\mathrm{t}) \mathrm{x}(\mathrm{t})+\mathrm{u}^{*^{\mathrm{T}}}(\mathrm{t}) \mathrm{G}(\mathrm{t}) \mathrm{u}^{*}(\mathrm{t})+\mathrm{A}^{\mathrm{u}^{*}} \mathrm{~h}^{*}(\mathrm{x})\right\}
\end{aligned}
$$

by equation (6) we have

$$
0 \leq x^{T}(t) C(t) x(t)+u^{* T}(t) G(t) u^{*}(t)+A^{u^{*}} h^{*}(x)
$$

Theorem (2). (covers of the HJB equation)

let $h^{*}(x)$ be a bounded function in $C(G)^{2} \cap C(C L(G))$, Suppouse that for all $u \in Y$ where $Y$ is the set of controlethe inequality

$$
x^{T}(t) C(t) x(t)+u^{T}(t) G(t) u(t)+A^{u} h^{*}(x) \geq 0
$$

then $\mathrm{h}^{*}(\mathrm{x}) \leq \mathrm{h}(\mathrm{x}, \mathrm{u})$, for all $\mathrm{u} \in \mathrm{Y}$, moreover

$x^{T}(t) C(t) x(t)+u^{* T}(t) G(t) u^{*}(t)+A^{u^{*}} h^{*}(x)=0$, Then

$\mathrm{u}^{*}$ is an optimal controle

Proof

Let $\mathrm{u}$ be a Markov control, and let $\mathrm{u}$ be a Markova control then

$$
A^{u} h^{*}(x) \geq-\left[x^{T}(t) C(t) x(t)+u^{T}(t) G(t) u(t)\right] \text { for } u \in Y
$$

by equation (21)

$$
\begin{gathered}
\left.E_{x}\left[h^{*}(x)\right)\right]=h(x)+E_{x} \int_{0}^{T} A^{u} h^{*}(x) d t \\
\geq h(x)-E_{x} \int_{0}^{T} x^{T}(t) C(t) x(t)+u^{*^{T}}(t) G(t) u^{*}(t) d t
\end{gathered}
$$

Thus

$h(x) \leq E_{x}\left[h^{*}(x)+\int_{0}^{T} x^{T}(t) C(t) x(t)+u^{* T}(t) G(t) u^{*}(t) d t\right]=h(x, u)$, then $\mathrm{u}^{*}$ is an optimal controle.

\section{Application 1 [Economics Model and It's Optimization [Fractional Stochastic Differential Equation]]}

In 1928 F. R Ramsy introduced an economics model describing the rate of change of capital $\mathrm{K}$ and labor $\mathrm{L}$ in a market by a system of ordinary differential equation with $\mathrm{P}$ and $\mathrm{C}$ being the production and consumption rates respectively the model is given by

$$
\frac{\mathrm{dk}(\mathrm{t})}{\mathrm{dt}}=\mathrm{p}(\mathrm{t})-\mathrm{C}(\mathrm{t}), \frac{\mathrm{dL}(\mathrm{t})}{\mathrm{dt}}=\mathrm{a}(\mathrm{t}) \mathrm{L}(\mathrm{t})
$$

Where $a(t)$ is the rate of growth Labor.

The production, capital and labor are related by the Cobb-Douglas formula. 


$$
\mathrm{p}(\mathrm{t})=\mathrm{Ak}(t)^{\alpha} \mathrm{L}(t)^{\beta}
$$

where $A, \alpha, \beta$ are some positive constant.

in certain the dependence of $\mathrm{P}$ on $\mathrm{K}$ and $\mathrm{L}$ is linear these mean $\alpha=\beta=1$ which will be our assumption throughout this section we shall also assume that the labor is constant, $\mathrm{L}(\mathrm{t})=$ $\mathrm{L}_{0}$; which is true for certain markets or relatively short time intervals of several years.

Therefore the production rate and the capital are related by $\mathrm{p}(\mathrm{t})=\mathrm{H}(\mathrm{t}) \mathrm{k}(\mathrm{t}) \cdot[1]$

Another important assumption we make is that the production rate is subject to small random disturbances i.e $\mathrm{p}(\mathrm{t})=\mathrm{H}(\mathrm{t}) \mathrm{k}(\mathrm{t})+\mathrm{b}(\mathrm{k}(\mathrm{t})) \mathrm{dB}^{\mathrm{H}}$. therefore

$$
\frac{d k(t)}{d t}=H(t) k(t)+b(k(t)) d B^{H}-C(t)
$$

Where $M(t)=-C(t)$

Which can be rewritten in the differential form as

$$
d k(t)=[H(t) k(t)+M(t)] d t+b(k(t)) d B^{H}
$$

Where $\mathrm{B}^{\mathrm{H}}$ is fractional Brownian motion $\mathrm{b}(\mathrm{k}(\mathrm{t}))$ is real function, characteristic of the noise.

Assume that M ( $\mathrm{t}$ ) can be controlled

$$
\mathrm{dk}(\mathrm{t})=[\mathrm{H}(\mathrm{t}) \mathrm{k}(\mathrm{t})+\mathrm{u}(\mathrm{k}(\mathrm{t})) \mathrm{M}(\mathrm{t})] \mathrm{dt}+\mathrm{b}(\mathrm{k}(\mathrm{t})) \mathrm{dB}^{\mathrm{H}}(30)
$$

Usually one wants to minimize the cost function let us choose the following cost function

$$
\begin{gathered}
h(x, u)=E\left(\left(x^{T}(T) R x(T)+\int_{0}^{T}\left(x^{T}(t) C(t) x(t)+\right.\right.\right. \\
\left.\left.u^{T}(t) G(t) u(t)\right) d t\right)
\end{gathered}
$$

The operator onh* $(x)=\left(x^{T}(T) R x(T)\right)$

$$
\begin{gathered}
A^{u} h^{*}(x)=\left[\frac{d}{d x}\left(\left(x^{T}(T) R x(T)\right)\right](H(t) x(t)+M(t) u(t))+\right. \\
\int_{0}^{t} \frac{d^{2}}{d^{2} x}\left(\left(x^{T}(T) R x(T)\right) b(q) b(p) \emptyset_{H}(p-q) d s .\right.
\end{gathered}
$$

Since

$F^{\prime}(\mathrm{x}(\mathrm{t})) \mathrm{a}(\mathrm{t})=\left[\frac{\mathrm{d}}{\mathrm{dx}}\left(\mathrm{x}(\mathrm{T})^{\mathrm{T}} \mathrm{R} \mathrm{x}(\mathrm{T})\right)\right](\mathrm{H}(\mathrm{t}) \mathrm{x}(\mathrm{t})+\mathrm{M}(\mathrm{t}) \mathrm{u}(\mathrm{t}))$

$$
\begin{gathered}
\int_{0}^{t} F^{\prime \prime}\left(\mathrm{x}(\mathrm{P}) \int_{0}^{P} \operatorname{Dq} a(\mathrm{t}) \mathrm{dtb}(\mathrm{p}) \emptyset_{H}(\mathrm{p}-\mathrm{q}) \mathrm{dt}=0\right. \\
\int_{0}^{t} F^{\prime \prime}(\mathrm{x}(p)) b(\mathrm{q}) b(\mathrm{p}) \emptyset_{\mathrm{H}}(\mathrm{p}-\mathrm{q}) \mathrm{dt}=\int_{0}^{\mathrm{t}} \frac{\mathrm{d}^{2}}{\mathrm{~d}^{2} \mathrm{x}}\left(\mathrm{x}(\mathrm{T})^{\mathrm{T}} \mathrm{R}\right. \\
\mathrm{x}(\mathrm{T})) \mathrm{b}(\mathrm{q}) \mathrm{b}(\mathrm{p}) \emptyset_{\mathrm{H}}(\mathrm{p}-\mathrm{q}) \mathrm{dt} .
\end{gathered}
$$

by Theorem (1)

$\min \left\{\mathrm{x}^{\mathrm{T}}(\mathrm{t}) \mathrm{c}(\mathrm{t}) \mathrm{x}(\mathrm{t})+\mathrm{u}^{\mathrm{T}}(\mathrm{t}) \mathrm{G}(\mathrm{t}) \mathrm{u}(\mathrm{t})+2 \mathrm{R} x(\mathrm{~T}) \mathrm{H}(\mathrm{t}) \mathrm{x}(\mathrm{t})+\right.$ $2 \mathrm{Rx}(\mathrm{T}) \mathrm{M}(\mathrm{t}) \mathrm{u}(\mathrm{t})+\int_{0}^{t} 2 \mathrm{Rx}(\mathrm{T}) \mathrm{b}(\mathrm{q}) \mathrm{b}(\mathrm{p}) \emptyset_{\mathrm{H}}(\mathrm{p}-\mathrm{q}) d \mathrm{t}=$ 0

by taking the derivative of two sides, one can get,

$$
\begin{gathered}
\frac{d}{d u}\left\{x^{T}(t) C(t) x(t)+u^{T}(t) G(t) u(t)+2 R x(T) H(t)\right. \\
x(t)+2 R x(T) M(t) u(t)+\int_{0}^{t} 2 R x(T) b(q) b(p) \emptyset_{H}(p- \\
q) d t=0 \\
2 G(t) u(t)+2 R x(T) M(t)=0 \\
u(t)=-\frac{R x(T) M(t)}{G(t)}
\end{gathered}
$$

Is optimal control for the linear-quadratic fractional Brownian motion differential equation and the optimal cost function is

$$
\begin{gathered}
h(x, u)=E\left(x(T)^{T} R x(T)+\int_{0}^{T}\left(x^{T}(t) C(t) x(t)+\right.\right. \\
\left.\left.\frac{(R x(T) M(t))^{2}}{G(t)}\right) d t\right)
\end{gathered}
$$

\section{Stratonovich Stochastic Differential Equation}

Leta $(x(t)), b(x(t))$ are continuous functionaldefined on the metric space $\mathrm{K}$, let the stochastic process $\mathrm{x}(\mathrm{t})$ satisfy the Stratonovich Stochastic Differential Equation

$$
d x(t)=\tilde{a}(x(t)) d t+b(x(t)) \circ d B(t)
$$

where

$$
\begin{array}{r}
\tilde{a}(x(t))=a(x(t))-\frac{1}{2} b(x(t)) \frac{d b(x(t))}{d x(t)}, \\
b(x(t)) \circ d B(t)=b(x(t)) d B(t)+\frac{1}{2} b(x(t)) \frac{d b(x(t))}{d x(t)},
\end{array}
$$

and $\mathrm{B}(\mathrm{t})$ is Brownian motion.

Let $H(t)$ be $n \times n$ matrices, $M(t)$ be $n \times k$ matrices, $b(t)$ be $\mathrm{n} \times \mathrm{m}$ matrices and thecontrolu (t) be $\mathrm{k} \times 1$ vector, let $\mathrm{a}(\mathrm{x}(\mathrm{t}))=\mathrm{H}(\mathrm{t}) \mathrm{x}(\mathrm{t})+\mathrm{M}(\mathrm{t}) \mathrm{u}(\mathrm{t})$ and $\mathrm{b}(\mathrm{x}(\mathrm{t}))=\mathrm{b}(\mathrm{t})$, then $(6$. 2) become

$$
\begin{gathered}
\tilde{a}(x(t))=H(t) x(t)+M(t) u(t)-\frac{1}{2} b(t) \frac{d b(t)}{d x(t)} \\
\text { And equation (30) become } b(t) \circ d B(t)=b(t) d B(t)+\frac{1}{2} b(t) \frac{d b(t)}{d x(t)}
\end{gathered}
$$

then from (31) and (32) the stochastic process x (t) in (28) satisfy the linear Stratonovich Stochastic Differential Equation

$$
d x(t)=H(t) x(t)+M(t) u(t)-\frac{1}{2} b(t) \frac{d b(t)}{d x(t)}+b(t) \circ d B(t)
$$

Remark (4), "The ITO Stratonovich Taylor Formula"

Let the stochastic process $\mathrm{x}(\mathrm{t})$ defined as 


$$
x(t)=x(0)+\int_{0}^{t} \tilde{a}\left(x(t) d t+\int_{0}^{t} b(t) d B(t)\right.
$$

where $\tilde{a}(\mathrm{x}(\mathrm{t})), \mathrm{b}(\mathrm{t}) \circ \mathrm{dB}(\mathrm{t})$ are defined in equation (31) and equation (32) respectively, anda $(\mathrm{x}(\mathrm{t})), \mathrm{b}(\mathrm{x}(\mathrm{t}))$ are continuous functionaldefined on the metric space $\mathrm{K}$, then $\mathrm{x}(\mathrm{t})$ satisfy the ITO Stratonovich Taylor Formula for $\mathrm{f}: \mathrm{R} \rightarrow \mathrm{R}$

$$
f(x(t))=f(x(0))+\int_{0}^{t} \tilde{a}(x(t)) \frac{d f(x(t))}{d x} d t+\int_{0}^{t} b(t) \frac{d f(x(t))}{d x} d B(t)
$$

by applying (6.4) and (6.5) 0n (6.8) to get the ITO Formula

$$
f(x(t))=f(x(0))+\int_{0}^{t}\left[(H(t) x(t)+M(t) u(t)) \frac{d f(x(t))}{d x}-\frac{1}{2} b(t) \frac{d b(x(t))}{d x(t)} \frac{d f(x(t))}{d x}\right] d t+\int_{0}^{t}\left[b(t) \frac{d f(x(t))}{d x} d B(t)+\frac{1}{2} b(t) \frac{d f(x(t))}{d x} \frac{d b(t)}{d x(t)}\right] d t
$$

by taking the derivative of two sides, one can get,

$$
d f(x(t))=\left[(H(t) x(t)+M(t) u(t)) \frac{d f(x(t))}{d x}-\frac{1}{2} b(t) \frac{d b(t)}{d x(t)} \frac{d f(x(t))}{d x}\right] d t+\left[b(t) \frac{d f(x(t))}{d x} d B(t)+\frac{1}{2} b(t) \frac{d f(x(t))}{d x} \frac{d b(t)}{d x(t)}\right] d t
$$

Remark (5)

By definition (15) The generator $\mathrm{A}^{\mathrm{u}}$ of anStratonovich Stochastic different equation is

$$
\left(A^{u} f\right)=(H(t) x(t)+M(t) u(t)) \frac{d f(x(t))}{d x}-\frac{1}{2} b(t) \frac{d b(t)}{d x(t)} \frac{d f(x(t))}{d x}+\frac{1}{2} b(t) \frac{d f(x(t))}{d x} \frac{d b(t)}{d x(t)}
$$

\subsection{The Martingle Problem}

If (30) is an Stratonovich Stochastic Differential Equation with generator $\mathrm{A}^{\mathrm{u}}$ and $\mathrm{f} \in C^{2}(\mathrm{R})$ then

$$
f(x(t))=f(x(0))+\int_{0}^{t} A^{u} d t+\int_{0}^{t} b(t) \frac{d f(x(t))}{d x} d B(t)(42)
$$

\subsection{Dynkin Formula for Fractional Stochastic Linear Quadratic Regulator Problem with Stratonovich Formula}

Let $h \in C^{2}(\mathrm{R}), \mathrm{C}(\mathrm{t})$ be the $\mathrm{n} \times \mathrm{n}$ matrices and $\mathrm{G}(\mathrm{t})$ be the $\mathrm{k} \times \mathrm{k}$ matrices, Note that from (34), we obtain the following Stratonovich formula for the function $\mathrm{h}(\mathrm{x}(\mathrm{t}))$ where $\mathrm{h}(\mathrm{x}(\mathrm{t}))$ defined as

$$
h(x(t))=x^{T}(t) C(t) x(t)+G(t)
$$

$h(x(t))=h(x(0))+\int_{0}^{t}\left[(H(t) x(t)+M(t) u(t)) \frac{d h(x(t))}{d x}-\frac{1}{2} b(t) \frac{d b(t)}{d x(t)}\right.$

$\left.\frac{d h(x(t))}{d x}\right] d t+\int_{0}^{t}\left[b(t) \frac{d h(x(t))}{d x} d B(t)+\frac{1}{2} b(t) \frac{d h(x(t))}{d x} \frac{d b(t)}{d x(t)}\right] d t(44)$

Let $\mathrm{T}$ be a stopping time for the stochastic processx $(\mathrm{t})$ such that

$\mathrm{E}\left(\int_{0}^{\mathrm{T}} \mathrm{A}^{\mathrm{u}} \mathrm{h}(\mathrm{x}(\mathrm{t})) \mathrm{dt}<\infty\right.$, by taking the expectation of two sides, one can get the following Dynkin formula

$$
\mathrm{E}(\mathrm{h}(\mathrm{x}(\mathrm{T})))=\mathrm{h}(\mathrm{x}(0))+\mathrm{E}\left[\int_{0}^{\mathrm{T}}[(\mathrm{H}(\mathrm{t}) \mathrm{x}(\mathrm{t})+\mathrm{M}(\mathrm{t}) \mathrm{u}(\mathrm{t}))\right.
$$

$$
\begin{gathered}
\frac{d \mathrm{~h}(\mathrm{x}(\mathrm{t}))}{d \mathrm{x}}-\frac{1}{2} \mathrm{~b}(\mathrm{t}) \frac{\mathrm{db}(\mathrm{t})}{\mathrm{dx}(\mathrm{t})} \frac{\mathrm{dh}(\mathrm{x}(\mathrm{t}))}{\mathrm{dx}}+ \\
\left.\frac{1}{2} \mathrm{~b}(\mathrm{t}) \frac{\mathrm{dh}(\mathrm{x}(\mathrm{t}))}{\mathrm{dx}} \frac{\mathrm{db}(\mathrm{t})}{\mathrm{dx}(\mathrm{t})}\right] \\
\mathrm{E}(\mathrm{h}(\mathrm{x}(\mathrm{T})))=\mathrm{h}(\mathrm{x}(0))+\mathrm{E}\left[\int_{0}^{\mathrm{T}} \mathrm{A}^{\mathrm{u}} \mathrm{h}(\mathrm{x}(\mathrm{t})) \mathrm{dt}\right](45)
\end{gathered}
$$

\subsection{The Fractional Stochastic Quadratic Regulator Optimal Problem}

Assume that the cost linear quadratic regulator function is

$$
\begin{array}{r}
h(x, u)=E\left(x(T){ }^{T} R x(T)+\int_{0}^{T}\left(x^{T}(t) C(t) x(t)+\right.\right. \\
\left.\left.u^{T}(t) G(t) u(t)\right) d t\right)
\end{array}
$$

where all of the coefficients $C(t)$ be the $n \times n$ matrics, $G(t)$ be the $\mathrm{k} \times \mathrm{k}$, the control $\mathrm{u}(\mathrm{t})$ be $\mathrm{k} \times 1$ vector, we assume thatC $(\mathrm{t})$ and Rare symmetric, non negative definite and $G(t)$ is symmetric positive definite and $\mathrm{T}$ is the final time of the solution $x(t)$ where $x(t)$ defined in (25) such that $E_{x}|T|<\infty$, the problem is to find the optimal control $\mathrm{u}^{*}(\mathrm{t})$ such that

$$
\mathrm{h}\left(\mathrm{x}, \mathrm{u}^{*}(\mathrm{t})\right)=\min \{\mathrm{h}(\mathrm{x}, \mathrm{u})\}
$$

\subsection{Hamilton-Jacobi-Bellman Equation for Quadratic Regulator Problem}

Let the optimal control $\mathrm{u}^{*}(\mathrm{t}) \in \mathrm{Y}$ where $\mathrm{Y}$ is the set of control then the generator in equation (35) become

$$
\left(A^{u^{*}} h\right)=\left(H(t) x(t)+M(t) u^{*}(t)\right) \frac{d h(x(t))}{d x}-\frac{1}{2} b(t) \frac{d b(t)}{d x(t)} \frac{d h(x(t))}{d x}+\frac{1}{2} b(t) \frac{d h(x(t))}{d x} \frac{d b(t)}{d x(t)}
$$

Theorem (3) "HJB equation"

$$
\operatorname{Defineh}^{*}(\mathrm{x})=\min \{\mathrm{h}(\mathrm{x}, \mathrm{u}): \mathrm{u}=\mathrm{u}(\mathrm{t})-\text { Markov control }\}
$$

Suppose that $h \in C^{2}(\mathrm{R})$ and the optimal controlu*exists Then

$$
\min \left\{\mathrm{x}^{\mathrm{T}}(\mathrm{t}) \mathrm{C}(\mathrm{t}) \mathrm{x}(\mathrm{t})+\mathrm{u}^{\mathrm{T}}(\mathrm{t}) \mathrm{G}(\mathrm{t}) \mathrm{u}(\mathrm{t})+\mathrm{A}^{\mathrm{u}} \mathrm{h}^{*}(\mathrm{x})\right\}=0
$$

where $G(t)$ be the $k \times k$ metrics, the control $u(t)$ be $k \times 1$ vector, and the generator $\mathrm{A}^{\mathrm{u}}$ is given in equation (42) and 


$$
\mathrm{h}^{*}(\mathrm{x})=\mathrm{x}(\mathrm{T}){ }^{\mathrm{T}} \mathrm{R} x(\mathrm{~T})
$$

The minim is a chivied whenu* is optimal. In other words

$$
x^{T}(t) C(t) x(t)+u^{* T}(t) G(t) u^{*}(t)+A^{u^{*}} h^{*}(x)=0
$$

Proof

Now proceed to prove (43), let $\alpha=\operatorname{Tv}$ be the first exit time of the solution x (t) by using (4) and (5)

$$
\begin{gathered}
\mathrm{E}_{\mathrm{x}}[\mathrm{h}(\mathrm{x}(\alpha), \mathrm{u})]=\mathrm{E}_{\mathrm{x}}\left[\mathrm{E}_{\mathrm{x}}\left[\int_{0}^{\mathrm{T}}\left(\mathrm{x}^{\mathrm{T}}(\mathrm{t}) \mathrm{C}(\mathrm{t}) \mathrm{x}(\mathrm{t})+\mathrm{u}^{\mathrm{T}}(\mathrm{t}) \mathrm{G}(\mathrm{t}) \mathrm{u}(\mathrm{t})\right) \mathrm{dt}+\mathrm{x}(\mathrm{T}){ }^{\mathrm{T}} \mathrm{Rx}(\mathrm{T})\right]\right. \\
=\mathrm{E}_{\mathrm{x}}\left[\mathrm{E}_{\mathrm{x}}\left[\theta_{\alpha} \int_{0}^{\mathrm{T}}\left(\mathrm{x}^{\mathrm{T}}(\mathrm{t}) \mathrm{C}(\mathrm{t}) \mathrm{x}(\mathrm{t})+\mathrm{u}^{\mathrm{T}}(\mathrm{t}) \mathrm{G}(\mathrm{t}) \mathrm{u}(\mathrm{t})\right) \mathrm{dt}+\mathrm{x}^{\mathrm{T}}(\mathrm{t}) \mathrm{Rx}(\mathrm{T}) / F_{\alpha}\right]\right. \\
=\mathrm{E}_{\mathrm{x}}\left[\int_{\alpha}^{\mathrm{T}}\left(\mathrm{x}^{\mathrm{T}}(\mathrm{t}) \mathrm{C}(\mathrm{t}) \mathrm{x}(\mathrm{t})+\mathrm{u}^{\mathrm{T}}(\mathrm{t}) \mathrm{G}(\mathrm{t}) \mathrm{u}(\mathrm{t})\right) d t\right. \\
\left.-\int_{0}^{\alpha}\left[\mathrm{x}^{\mathrm{T}}(\mathrm{t}) \mathrm{C}(\mathrm{t}) \mathrm{x}(\mathrm{t})+\mathrm{u}^{\mathrm{T}}(\mathrm{t}) \mathrm{G}(\mathrm{t}) \mathrm{u}(\mathrm{t})\right] \mathrm{dt}\right]
\end{gathered}
$$

$E_{x}[h(x, u)]=h(x, u)-E_{x}\left[\int_{0}^{\alpha}\left(x^{T}(t) C(t) x(t)+u^{T}(t) G(t) u(t)\right) d t\right]$, Thus

$$
\begin{array}{r}
h(x, u)=E_{x}\left[\int_{0}^{\alpha} x^{T}(t) C(t) x(t)+u^{T}(t) G(t) u(t) d t\right]+E_{x}[h(x, u)](53) \\
h^{*}(x) \leq h(x, u)=E_{x}\left[\int_{0}^{\alpha} x^{T}(t) C(t) x(t)+u^{T}(t) G(t) u(t) d s\right]+E_{x}[h(x, u)]
\end{array}
$$

by equation (37)

$$
\begin{gathered}
E_{x}[h(x, u)]=h(x)+E_{x} \int_{0}^{\alpha} A^{u^{*}} h^{*}(x) d t \\
h^{*}(x) \leq h(x, u)=E_{x}\left[\int_{0}^{\alpha} x^{T}(t) C(t) x(t)+u^{T}(t) G(t) u(t) d t\right]+h(x)+E_{x} \int_{0}^{\alpha} A^{u^{*}} h^{*}(x) d t
\end{gathered}
$$

Or $0 \leq E_{x}\left[\int_{0}^{\alpha} x^{T}(t) C(t) x(t)+u^{T}(t) G(t) u(t) d s\right]+E_{x} \int_{0}^{\alpha} A^{u^{*}} h^{*}(x) d t$

At $\alpha \rightarrow 0$. Thus $0 \leq \mathrm{E}_{\mathrm{x}}\left\{\mathrm{x}^{\mathrm{T}}(\mathrm{t}) \mathrm{C}(\mathrm{t}) \mathrm{x}(\mathrm{t})+\mathrm{u}^{*^{\mathrm{T}}}(\mathrm{t}) \mathrm{G}(\mathrm{t}) \mathrm{u}^{*}(\mathrm{t})+\mathrm{A}^{\mathrm{u}^{*}} \mathrm{~h}^{*}(\mathrm{x})\right\}$

by (37) we have

$$
0 \leq x^{T}(t) C(t) x(t)+u^{* T}(t) G(t) u^{*}(t)+A^{u^{*}} h^{*}(x)
$$

Theorem (4). (convers of the HJB_equation)

let $\mathrm{h}^{*}(\mathrm{x})$ be a bounded function in $C(G)^{2} \cap \mathrm{C}(\mathrm{CL}(\mathrm{G}))$, Suppose that for all $\mathrm{u} \in \mathrm{Y}$ where $\mathrm{Y}$ is the set of control the inequality

$$
x^{T}(t) C(t) x(t)+u^{T}(t) G(t) u(t)+A^{u} h^{*}(x) \geq 0
$$

then $\mathrm{h}^{*}(\mathrm{x}) \leq \mathrm{h}(\mathrm{x}, \mathrm{u})$, for all $\mathrm{u} \in \mathrm{Y}$, moreover

$x^{T}(t) C(t) x(t)+u^{*}{ }^{*}(t) G(t) u^{*}(t)+A^{u^{*}} h^{*}(x)=0$, Then $\mathrm{u}^{*}$ is an optimal controle

\section{Proof}

Let $\mathrm{u}$ be a Markov control, and let $\mathrm{u}$ be a Markova control then

$$
A^{u} h^{*}(x) \geq-x^{T}(t) C(t) x(t)+u^{T}(t) G(t) u(t) \text { for } u \in Y
$$

by equation (37)

$$
\begin{gathered}
\left.E_{x}\left[h^{*}(x)\right)\right]=h(x)+E_{x} \int_{0}^{T} A^{u} h^{*}(x) d t \\
\geq h(x)-E_{x} \int_{0}^{T} x^{T}(t) C(t) x(t)+u^{* T}(t) G(t) u^{*}(t) d t
\end{gathered}
$$

Thus

$$
h(x) \leq E_{x}\left[h^{*}(x)+\int_{0}^{T} x^{T}(t) C(t) x(t)+\right.
$$

$$
\left.u^{*^{T}}(t) G(t) u^{*}(t) d t\right]=h(x, u)
$$

therefore

$\mathrm{u}^{*}$ is an optimal controle.

\section{Application 2 [Economics Model with Brownian Stronovich Differential Equation]}

In 1928 F. R Ramsy introduced an economics model describing the rate of change of capital $\mathrm{K}$ and labor $\mathrm{L}$ in a market by a system of ordinary differential equation with $\mathrm{P}$ and $\mathrm{C}$ being the production and consumption rates respectively the model is given by

$$
\frac{\mathrm{dk}(\mathrm{t})}{\mathrm{dt}}=\mathrm{p}(\mathrm{t})-\mathrm{C}(\mathrm{t}), \frac{\mathrm{dL}(\mathrm{t})}{\mathrm{dt}}=\mathrm{a}(\mathrm{t}) \mathrm{L}(\mathrm{t})
$$

Where $a(t)$ is the rate of growth Labor.

The production, capital and labor are related by the Cobb-Douglas formula.

$$
\mathrm{p}(\mathrm{t})=\mathrm{Ak}(\mathrm{t})^{\alpha} \mathrm{L}(\mathrm{t})^{\beta}
$$

where $A, \alpha, \beta$ are some positive constant. in certain the 
dependence of $\mathrm{P}$ on $\mathrm{K}$ and $\mathrm{L}$ is linear these mean $\alpha=\beta=$ 1 which will be our assumption throughout this section we shall also assume that the labor is constant, $\mathrm{L}(\mathrm{t})=\mathrm{L}_{0}$; which is true for certain markets or relatively short time intervals of several years.

Therefore the production rate and the capital are related by

$$
p(t)=H(t) k(t) \cdot[1]
$$

A nether important assumption we make is that the production rate is subject to small random disturbances i.e $p(t)=H(t) k(t)+b(k(t)) \circ d B(t)$. therefore

$$
\frac{d k(t)}{d t}=H(t) k(t)+b(k(t)) \circ d B(t)-C(t)
$$

Where $\mathrm{M}(\mathrm{t})=-\mathrm{C}(\mathrm{t})$

Which can be rewritten in the differential form as

$$
d k(t)=[H(t) k(t)+M(t)] d t+b(k(t)) \circ d B(t)(55)
$$

Where $B(t)$ is Brownian motion $b(k(t))$ is real function, characteristic of the noise, Assume that $M(t)$ can be controlled then equation (55) become

$$
\mathrm{dk}(\mathrm{t})=[\mathrm{H}(\mathrm{t}) \mathrm{k}(\mathrm{t})+\mathrm{M}(\mathrm{t}) \mathrm{u}(\mathrm{k}(\mathrm{t}))] \mathrm{dt}+\mathrm{b}(\mathrm{k}(\mathrm{t})) \circ \mathrm{dB}(\mathrm{t})(56)
$$

usually one wants to minimize the cost function (38) let $\mathrm{h}^{*}$ $(\mathrm{x})=\mathrm{x}^{\mathrm{T}}(\mathrm{T}) \mathrm{R} \mathrm{x}(\mathrm{T})$, and let $\mathrm{h}^{*}(\mathrm{x}(\mathrm{T})) \in \mathrm{D}\left(\mathrm{A}^{\mathrm{u}}\right)$ and then $\left(A^{u} h^{*}\right)$ is

$$
\begin{gathered}
\left(\mathrm{A}^{\mathrm{u}} \mathrm{h}^{*}\right) \mathrm{x}=(\mathrm{H}(\mathrm{t}) \mathrm{x}(\mathrm{t})+\mathrm{M}(\mathrm{t}) \mathrm{u}(\mathrm{t})) \frac{\mathrm{dh}^{*}(\mathrm{x}(\mathrm{T}))}{\mathrm{dx}}-\frac{1}{2} \mathrm{~b}(\mathrm{k}(\mathrm{t})) \\
\frac{\mathrm{db}(\mathrm{k}(\mathrm{t}))}{\mathrm{dk}(\mathrm{t})} \frac{\mathrm{dh}^{*}(\mathrm{x}(\mathrm{T}))}{\mathrm{dx}}+ \\
\frac{1}{2} \mathrm{~b}(\mathrm{k}(\mathrm{t})) \frac{\mathrm{dh}^{*}(\mathrm{x}(\mathrm{t}))}{\mathrm{dx}} \frac{\mathrm{db}(\mathrm{k}(\mathrm{t}))}{\mathrm{dk}(\mathrm{t})}
\end{gathered}
$$

$\left(\mathrm{A}^{\mathrm{u}} \mathrm{h}^{*}\right)=\mathrm{H}(\mathrm{t}) \mathrm{x}(\mathrm{t}) 2 \mathrm{Rx}(\mathrm{t})+\mathrm{M}(\mathrm{t}) \mathrm{u}(\mathrm{t}) 2 \mathrm{Rx}(\mathrm{t})-\mathrm{b}(\mathrm{k}(\mathrm{t}))$

$$
\frac{\mathrm{db}(\mathrm{k}(\mathrm{t}))}{\mathrm{dk}(\mathrm{t})} \mathrm{R} \times(\mathrm{T})+\mathrm{b}(\mathrm{k}(\mathrm{t})) \mathrm{R} \times(\mathrm{T}) \frac{\mathrm{db}(\mathrm{k}(\mathrm{t}))}{\mathrm{dk}(\mathrm{t})}
$$

Then equation (43) becomeh* $(\mathrm{x})+\left(\mathrm{A}^{\mathrm{u}^{*}} \mathrm{~h}^{*}\right)=0$

$$
\begin{gathered}
x^{T}(t) C(t) x(t)+u^{T}(t) G(t) u(t)+H(t) x(t) 2 R x(t)+M(t) \\
u(t) 2 R x(t)-b(k(t)) \frac{d b(k(t))}{d k(t)} R x(T)+b(k(t)) R x(T) \\
\frac{d b(k(t))}{d k(t)}=0
\end{gathered}
$$

bytaking the derivative of two sides, one can get,

$$
\begin{gathered}
\frac{d}{d u}\left[x^{T}(t) C(t) x(t)+u^{T}(t) G(t) u(t)+H(t) x(t) 2 R x(T)+\right. \\
M(t) u(t) 2 R x(T)-b(k(t)) \frac{d b(k(t))}{d k(t)} R x(T)+b(k(t)) R x \\
\left.(T) \frac{d b(k(t))}{d k(t)}\right]=0 \\
2 u(t) G(t)+M(t) 2 R x(t)=0 \\
u(t)=-\frac{M(t) R x(T)}{G(t)}
\end{gathered}
$$

is an optimal control for stratonovich stochastic linear quadratic differential equation and the optimal cost function is

$$
\begin{gathered}
h(x, u)=E\left(x(T)^{T} R x(T)+\int_{0}^{T}\left(x^{T}(t) C(t) x(t)+\right.\right. \\
\left.\left.\frac{(M(t) R x(T))^{2}}{G(t)}\right) d t\right)
\end{gathered}
$$

\section{Fractional Stratonovich Stochastic Differential Equation}

Let $\mathrm{a}(\mathrm{x}(\mathrm{t})), \mathrm{b}(\mathrm{x}(\mathrm{t}))$ are continuous functionaldefined on the metric space $K$, let the Fractional stochastic process $x(t)$ satisfy the Fractional Stratonovich Stochastic Differential Equation

$$
d x(t)=\tilde{a}(x(t)) d t+b(x(t)) \circ d^{H}(t)
$$

$$
\text { where } \tilde{a}(x(t))=a(x(t))-\frac{1}{2} b(x(t)) \frac{d b(x(t))}{d x(t)} \text {, }
$$

$\mathrm{b}(\mathrm{x}(\mathrm{t})) \circ \mathrm{dB}^{\mathrm{H}}(\mathrm{t})=\mathrm{b}(\mathrm{x}(\mathrm{t})) \mathrm{dB}^{\mathrm{H}}(\mathrm{t})+\frac{1}{2} \mathrm{~b}(\mathrm{x}(\mathrm{t})) \frac{\mathrm{db}(\mathrm{x}(\mathrm{t}))}{\mathrm{dx}(\mathrm{t})}$,

and $\mathrm{B}^{\mathrm{H}}$ (t) Fractional is Brownian motion.

Let $H(t)$ be $n \times n$ matrices, $M(t)$ be $n \times k$ matrices, $b(t)$ be $\mathrm{n} \times \mathrm{m}$ matrices and thecontrol $\mathrm{u}(\mathrm{t})$ be $\mathrm{k} \times 1$ vector, let $\mathrm{a}(\mathrm{x}(\mathrm{t}))=\mathrm{H}(\mathrm{t}) \mathrm{x}(\mathrm{t})+\mathrm{M}(\mathrm{t}) \mathrm{u}(\mathrm{t})$ and $\mathrm{b}(\mathrm{x}(\mathrm{t}))=\mathrm{b}(\mathrm{t})$, then (34) become

$$
\tilde{a}(x(t))=H(t) x(t)+M(t) u(t)-\frac{1}{2} b(t) \frac{d b(t)}{d x(t)}
$$

and equation (35) become

$$
b(t) \circ d B^{H}(t)=b(t) d B^{H}(t)+\frac{1}{2} b(t) \frac{d b(t)}{d x(t)}
$$

then from equation (60) and equation (61) the Fractional stochastic process $x(t)$ in equation (57) satisfy the Fractional linear Stratonovich Stochastic Differential Equation

$$
d x(t)=H(t) x(t)+M(t) u(t)-\frac{1}{2} b(t) \frac{d b(t)}{d x}+b(t) \circ d B^{H}(t)
$$

Remark (6), "The ITO Fractional Stratonovich Taylor Formula"

Let the stochastic process $\mathrm{x}(\mathrm{t})$ defined as

$$
x(t)=x(0)+\int_{0}^{t} \tilde{a}\left(x(t) d t+\int_{0}^{t} b(t) d B^{H}(t)\right.
$$

where $\tilde{a}(\mathrm{x}(\mathrm{t})), \mathrm{b}(\mathrm{t}) \circ \mathrm{dB}^{\mathrm{H}}(\mathrm{t})$ are defined in equation (58) and equation (59) respectively, and $\mathrm{a}(\mathrm{x}(\mathrm{t})), \mathrm{b}(\mathrm{x}(\mathrm{t}))$ are continuous functionaldefined on the metric space $K$, then $x$ (t) satisfy the ITO Fractional Stratonovich Taylor Formula for f: $R \rightarrow R$

$$
\begin{aligned}
& \mathrm{f}(\mathrm{x}(\mathrm{t}))=\mathrm{f}(\mathrm{x}(0))+ \\
& \int_{0}^{t}\left(\frac{d f(x(t))}{d x} \tilde{a}(x(t)) d t+\int_{0}^{t} \int_{0}^{t}\left(\frac{d^{2} f(x(p))}{d^{2} x}\right) \int_{0}^{p} D_{q}(\tilde{a}(x(t)) d t b(p)\right. \\
& \emptyset_{\mathrm{H}}(\mathrm{p}-\mathrm{q}) \mathrm{dqdp}+\int_{0}^{\mathrm{t}} \int_{0}^{\mathrm{p}}\left(\frac{d^{2} \mathrm{f}(\mathrm{x}(\mathrm{p}))}{d^{2} \mathrm{x}} \mathrm{b}(\mathrm{q}) \mathrm{b}(\mathrm{p}) \emptyset_{\mathrm{H}}(\mathrm{p}-\mathrm{q}) \mathrm{dpdq}+\right. \\
& \int_{0}^{t}\left(\frac{d f(x(t))}{d x} b(t) d B^{H}(t)\right)+\int_{0}^{t} \int_{0}^{t}\left(\frac{d^{2} f(x(p))}{d^{2} x} \int_{0}^{p} D_{q}(b(t)) d B^{H}(t) b(p)\right. \\
& \emptyset_{\mathrm{H}}(\mathrm{p}-\mathrm{q}) \mathrm{dqdp}
\end{aligned}
$$

by applying equation (58) and equation (59) on equation (64) 
to get the ITO Formula

$$
\begin{gathered}
f(x(t))=f(x(0))+\int_{0}^{t} \frac{d f(x(t))}{d x}(H(t) x(t)+M(t) u(t)) \\
-\frac{d f(x(t))}{d x} \frac{1}{2} b(t) \\
\left.\frac{d b(t)}{d x}\right] d t+\int_{0}^{t} \int_{0}^{t} \frac{d^{2} f(x(p))}{d^{2} x} \int_{0}^{p} D_{q}\left[H(t) x(t)+M(t) u(t)-\frac{1}{2} b\right. \\
\left.(t) \frac{d b(t)}{d x}\right] d t b(p)
\end{gathered}
$$

$\emptyset_{\mathrm{H}}(\mathrm{p}-\mathrm{q}) \mathrm{dqdp}+\int_{0}^{\mathrm{t}} \int_{0}^{\mathrm{p}}\left(\frac{\mathrm{d}^{2} \mathrm{f}(\mathrm{x}(\mathrm{p}))}{\mathrm{d}^{2} \mathrm{x}} \mathrm{b}(\mathrm{q}) \mathrm{b}(\mathrm{p}) \emptyset_{\mathrm{H}}(\mathrm{p}-\mathrm{q}) \mathrm{dpdt}\right.$

$$
\begin{gathered}
+\int_{0}^{\mathrm{t}}\left[\frac{\mathrm{df}(\mathrm{x}(\mathrm{t}))}{\mathrm{dx}} \mathrm{b}(\mathrm{t}) \mathrm{dB}^{\mathrm{H}}(\mathrm{t})+\frac{1}{2} \mathrm{~b}(\mathrm{t}) \frac{\mathrm{df}(\mathrm{x}(\mathrm{t}))}{\mathrm{dx}} \frac{\mathrm{d}^{2} \mathrm{f}(\mathrm{x}(\mathrm{t}))}{\mathrm{d}^{2} \mathrm{x}} \frac{\mathrm{db}(\mathrm{t})}{\mathrm{dx}}\right] \mathrm{dt} \\
+\int_{0}^{\mathrm{t}} \int_{0}^{\mathrm{t}} \frac{\mathrm{d}^{2} \mathrm{f}(\mathrm{x}(\mathrm{p}))}{\mathrm{d}^{2} \mathrm{x}} \int_{0}^{\mathrm{p}}\left[\mathrm{D}_{q}(\mathrm{~b}(\mathrm{t})) \mathrm{dB}^{\mathrm{H}}(\mathrm{t}) \mathrm{b}(\mathrm{p}) \emptyset_{\mathrm{H}}(\mathrm{p}-\mathrm{q})\right. \\
+\frac{1}{2} \mathrm{D}_{\mathrm{q}}(\mathrm{b}(\mathrm{t}))\left(\mathrm{D}_{\mathrm{q}}^{2}(\mathrm{~b}(\mathrm{t}))\right] \mathrm{dqdqdt}
\end{gathered}
$$

By taking the derivative of two sides, one can get,

$$
\begin{gathered}
d f(x(t))=\left[\frac{d f(x(t))}{d x}(H(t) x(t)+M(t) u(t))-\frac{d f(x(t))}{d x} \frac{1}{2} b(t)\right. \\
\left.\frac{d b(t)}{d x(t)}\right] d t+\int_{0}^{t} \frac{d^{2} f(x(p))}{d^{2} x} \int_{0}^{p} D_{q} \\
{\left[H(t) x(t)+M(t) u(t)-\frac{1}{2} b(t) \frac{d b(x(t))}{d x}\right] d t b(p) \emptyset_{H}(p-q)} \\
\int_{0}^{p}\left(\frac{d^{2} f(x(p))}{d^{2} x} b(q) b(p) \emptyset_{H}(p-q) d p d t+\frac{d f(x(t))}{d x} b(t)\right. \\
d B^{H}(t) d t+\frac{1}{2} b(t) \frac{d f(x(t))}{d x} \\
\frac{d^{2} f(x(t))}{d^{2} x} \frac{d b(t)}{d x} d t+\int_{0}^{t} \frac{d^{2} f(x(p))}{d^{2} x} \int_{0}^{p}\left[D_{q}(b(t)) d B^{H}(t) b(p) \emptyset_{H}\right. \\
(p-q)+\frac{1}{2} D_{q}(b(t))\left(D^{2}{ }_{q}(b(t))\right] d q d t
\end{gathered}
$$

\section{Remark (7)}

by using substitution equation (66) in equation (16) one get that

$$
\begin{gathered}
\left(A^{\mathrm{u}} \mathrm{f}\right)=\frac{\mathrm{df}(\mathrm{x}(\mathrm{t}))}{\mathrm{dx}}(\mathrm{H}(\mathrm{t}) \mathrm{x}(\mathrm{t})+\mathrm{M}(\mathrm{t}) \mathrm{u}(\mathrm{t}))-\frac{\mathrm{df}(\mathrm{x}(\mathrm{t}))}{\mathrm{dx}} \frac{1}{2} \mathrm{~b}(\mathrm{t}) \\
\frac{\mathrm{db}(\mathrm{t})}{\mathrm{dx}(\mathrm{t})}+\int_{0}^{\mathrm{t}} \frac{\mathrm{d}^{2} \mathrm{f}(\mathrm{x}(\mathrm{p}))}{\mathrm{d}^{2} \mathrm{x}} \int_{0}^{\mathrm{p}} \mathrm{D}_{\mathrm{q}}
\end{gathered}
$$$$
\left[H(t) x(t)+M(t) u(t)-\frac{1}{2} b(t) \frac{d b(x(t))}{d x}\right] d t b(p) \emptyset_{H}(p-q)
$$$$
\int_{0}^{\mathrm{p}}\left(\frac{\mathrm{d}^{2} \mathrm{f}(\mathrm{x}(\mathrm{p}))}{d^{2} \mathrm{x}} \mathrm{b}(\mathrm{q}) \mathrm{b}(\mathrm{p}) \emptyset_{\mathrm{H}}(\mathrm{p}-\mathrm{q}) \mathrm{dp}+\frac{1}{2} \mathrm{~b}(\mathrm{t})\right.
$$$$
\frac{d f(x(t))}{d x} \frac{d^{2} f(x(t))}{d^{2} x} \frac{d b(t)}{d x}+\frac{1}{2} D_{q}(b(t))\left(D^{2}{ }_{q}(b(t))\right] d q
$$

Let $h \in C^{2}(\mathrm{R}), C(\mathrm{t})$ be the $\mathrm{n} \times \mathrm{n}$ matrices and $\mathrm{G}(\mathrm{t})$ be the $\mathrm{k} \times \mathrm{k}$ matrices, Note that from equation (33) and equation (34) we obtain the following Stratonovich formula for the function $\mathrm{h}(\mathrm{x}(\mathrm{t}))$ where $\mathrm{h}(\mathrm{x}(\mathrm{t}))$ defined as

$$
\mathrm{h}(\mathrm{x}(\mathrm{t}))=\mathrm{x}^{\mathrm{T}}(\mathrm{t}) \mathrm{C}(\mathrm{t}) \mathrm{x}(\mathrm{t})+\mathrm{G}(\mathrm{t})
$$

$\mathrm{h}(\mathrm{x}(\mathrm{t}))=\mathrm{h}(\mathrm{x}(0))+\int_{0}^{\mathrm{t}}[(2 \mathrm{C}(\mathrm{t}) \mathrm{x}(\mathrm{t}) \mathrm{H}(\mathrm{t}) \mathrm{x}(\mathrm{t})+2 \mathrm{C}(\mathrm{t}) \mathrm{x}(\mathrm{t})$ $M(t) u(t)) d t+\int_{0}^{t} \int_{0}^{p} 2 C(t) b(q) b(p) \emptyset_{H}(p-q) d q d t+\int_{0}^{t} 2 C$

$$
\text { (t) } x(t) b(t) d B^{H}
$$

Then equation (35) become

$$
\begin{gathered}
\left(A^{u} h\right)=2 C(t) x(t) H(t) x(t)+2 C(t) x(t) M(t) u(t)+\int_{0}^{p} 2 C \\
(t) b(q) b(p) \emptyset_{H}(p-q) d q
\end{gathered}
$$

\subsection{The Fractional Stratonovich Martingle Problem}

If (62) is an ITO Fractional Stratonovich Stochastic Differential Equation withgenerator $\mathrm{A}^{\mathrm{u}}$ and $\mathrm{f} \in C^{2}(\mathrm{R})$ then

$$
\begin{gathered}
\mathrm{f}(\mathrm{x}(\mathrm{t}))=\mathrm{f}(\mathrm{x}(0))+\int_{0}^{\mathrm{t}} \mathrm{A}^{\mathrm{u}} \mathrm{fdt}+\int_{0}^{\mathrm{t}}\left[\frac{\mathrm{df}(\mathrm{x}(\mathrm{t}))}{\mathrm{dx}} \mathrm{b}(\mathrm{t}) \mathrm{dB}^{\mathrm{H}}(\mathrm{t})+\frac{1}{2} \mathrm{~b}(\mathrm{t})\right. \\
\left.\frac{\mathrm{df}(\mathrm{x}(\mathrm{t}))}{\mathrm{dx}} \frac{\mathrm{d}^{2} \mathrm{f}(\mathrm{x}(\mathrm{t}))}{\mathrm{d}^{2} \mathrm{x}} \frac{\mathrm{db}(\mathrm{t})}{\mathrm{dx}}\right] \mathrm{dt}+ \\
\int_{0}^{\mathrm{t}} \int_{0}^{\mathrm{t}} \frac{\mathrm{d}^{2} \mathrm{f}(\mathrm{x}(\mathrm{p}))}{\mathrm{d}^{2} \mathrm{x}} \int_{0}^{\mathrm{p}}\left[\mathrm{D}_{q}(\mathrm{~b}(\mathrm{t})) \mathrm{dB}^{\mathrm{H}}(\mathrm{t}) \mathrm{b}(\mathrm{p}) \emptyset_{\mathrm{H}}(\mathrm{p}-\mathrm{q})+\right. \\
\frac{1}{2} \mathrm{D}_{\mathrm{q}}(\mathrm{b}(\mathrm{t}))\left(\mathrm{D}_{\mathrm{q}}^{2}(\mathrm{~b}(\mathrm{t}))\right] \mathrm{dqdqdt}
\end{gathered}
$$

\subsection{Dynkin Formula for the Fractional Linear Stratonovich Quadratic Regulator Problem}

Let $h \in C^{2}(\mathrm{R}), \mathrm{C}(\mathrm{t})$ be the $\mathrm{n} \times \mathrm{n}$ matrices and $\mathrm{G}(\mathrm{t})$ be the $\mathrm{k} \times \mathrm{k}$ matrices, Note that from equation (33) and equation (34) we obtain the following Stratonovich formula for the function $\mathrm{h}(\mathrm{x}(\mathrm{t}))$ where $\mathrm{h}(\mathrm{x}(\mathrm{t}))$ defined as

$$
h(x(t))=x^{T}(t) C(t) x(t)+G(t)
$$

$\mathrm{h}(\mathrm{x}(\mathrm{t}))=\mathrm{h}(\mathrm{x}(0))+\int_{0}^{\mathrm{t}}[(2 \mathrm{C}(\mathrm{t}) \mathrm{x}(\mathrm{t}) \mathrm{H}(\mathrm{t}) \mathrm{x}(\mathrm{t})+2 \mathrm{C}(\mathrm{t}) \mathrm{x}(\mathrm{t})$ $M(t) u(t)] d t+\int_{0}^{t} \int_{0}^{p} 2 C(t) b(q) b(p) \emptyset_{H}(p-q) d q d t+\int_{0}^{t} 2 C$

$$
\text { (t) } x(t) b(t) d B^{H}
$$

Let $\mathrm{T}$ be a stopping time for the stochastic processx $(\mathrm{t})$ such that

$\mathrm{E}\left(\int_{0}^{\mathrm{T}} \mathrm{A}^{\mathrm{u}} \mathrm{h}(\mathrm{x}(\mathrm{t})) \mathrm{dt}<\infty\right.$, by taking the expectation of two sides, one can get the following Dynkin formula

$$
\begin{gathered}
E(h(x(T)))=h(x(0))+E\left[\int_{0}^{T}[2 C(t) x(t) H(t) x(t)+2 C(t)\right. \\
x(t) M(t) u(t) \\
\left.+\int_{0}^{p} 2 C(t) b(q) b(p) \emptyset_{H}(p-q)\right] d q d t \\
E(h(x(T)))=h(x(0))+E\left[\int_{0}^{T} A^{u} h(x(t)) d t\right]
\end{gathered}
$$

\subsection{The Fractional Stochastic Quadratic Regulator Optimal Problem with Stratonovich}

We assume that the cost linear quadratic regulator function is

$$
\begin{array}{r}
h(x, u)=E\left(x(T)^{T} R x(T)+\int_{0}^{T}\left(x^{T}(t) C(t) x(t)+\right.\right. \\
\left.\left.u^{T}(t) G(t) u(t)\right) d t\right)
\end{array}
$$

where all of the coefficients $C(t)$ be the $n \times n$ matrics, $G(t)$ be the $k \times k$, the control $u(t)$ be $k \times 1$ vector, we assume thatC $(t)$ and Rare symmetric, non negative definite and $G(t)$ is symmetric positive definite and $\mathrm{T}$ is the final time of the solution $x(t)$ where $x(t)$ defined in(12.7) such that $E_{x}|T|$ 
$<\infty$, the problem is to find the optimal control $\mathrm{u}^{*}(\mathrm{t})$ such that

$$
\mathrm{h}\left(\mathrm{x}, \mathrm{u}^{*}(\mathrm{t})\right)=\min \{\mathrm{h}(\mathrm{x}, \mathrm{u})\}
$$

\section{Hamilton-Jacobi-Bellman Equation for Fractional Stochastic Quadratic Regulator Problem}

Let the optimal control $u^{*}(t) \in Y$ where $Y$ is the set of control then the generator in equation (16) become

$$
\begin{aligned}
\left(\mathrm{A}^{\mathrm{u}^{*}} \mathrm{~h}\right)= & 2 \mathrm{C}(\mathrm{t}) \times(\mathrm{t}) \mathrm{H}(\mathrm{t}) \times(\mathrm{t})+2 \mathrm{C}(\mathrm{t}) \times(\mathrm{t}) \mathrm{M}(\mathrm{t}) \mathrm{u}^{*}(\mathrm{t}) \\
& +\int_{0}^{\mathrm{p}} 2 \mathrm{C}(\mathrm{t}) \mathrm{b}(\mathrm{q}) \mathrm{b}(\mathrm{p}) \emptyset_{\mathrm{H}}(\mathrm{p}-\mathrm{q}) d \mathrm{q}
\end{aligned}
$$

\section{Theorem (5) "HJB equation "}

$\operatorname{Defineh}^{*}(\mathrm{x})=\min \{\mathrm{h}(\mathrm{x}, \mathrm{u}): \mathrm{u}=\mathrm{u}(\mathrm{t})-$ Markov control $\}$ (78)

Suppose thath $\in C^{2}(\mathrm{R})$ and the optimal controlu* exists Then

$$
\min \left\{\mathrm{x}^{\mathrm{T}}(\mathrm{t}) \mathrm{C}(\mathrm{t}) \mathrm{x}(\mathrm{t})+\mathrm{u}^{\mathrm{T}}(\mathrm{t}) \mathrm{G}(\mathrm{t}) \mathrm{u}(\mathrm{t})+\mathrm{A}^{\mathrm{u}} \mathrm{h}^{*}(\mathrm{x})\right\}=0
$$

where $G(t)$ be the $k \times k$ metrics, the control $u(t)$ be $k \times 1$ vector, and the generator $\mathrm{A}^{\mathrm{u}}$ is given by equation (77) and

$$
\mathrm{h}^{*}(\mathrm{x})=\mathrm{x}(\mathrm{T}){ }^{\mathrm{T}} \mathrm{R} \mathrm{x}(\mathrm{T})
$$

The minim is a chivied whenu* is optimal. In other words

$$
x^{T}(t) C(t) x(t)+u^{*}(t) G(t) u^{*}(t)+A^{u^{*}} h^{*}(x)=0
$$

Proof

Now proceed to prove equation (81), let $\alpha=\mathrm{Tv}$ be the first exit time of the solution $\mathrm{x}(\mathrm{t})$ by using (4) and equation (5)

$$
\begin{gathered}
E_{x}[h(x(\alpha), u)]=E_{x}\left[E _ { x } \left[\int _ { 0 } ^ { T } \left(x^{T}(t) C(t) x(t)+u^{T}(t) G(t)\right.\right.\right. \\
\left.u(t)) d t+x(T)^{T} R x(T)\right]
\end{gathered}
$$

$\mathrm{E}_{\mathrm{x}}[\mathrm{h}(\mathrm{x}, \mathrm{u})]=\mathrm{h}(\mathrm{x}, \mathrm{u})-\mathrm{E}_{\mathrm{x}}\left[\int_{0}^{\alpha}\left(\mathrm{x}^{\mathrm{T}}(\mathrm{t}) \mathrm{C}(\mathrm{t}) \mathrm{x}(\mathrm{t})+\right.\right.$ $\left.\left.u^{T}(t) G(t) u(t)\right) d t\right]$, Thus

$$
\begin{gathered}
h(x, u)=E_{x}\left[\int_{0}^{\alpha} x^{T}(t) C(t) x(t)+u^{T}(t) G(t) u(t) d t\right]+ \\
E_{x}[h(x, u)] \\
h^{*}(x) \leq h(x, u)= \\
E_{x}\left[\int_{0}^{\alpha} x^{T}(t) C(t) x(t)+u^{T}(t) G(t) u(t) d s\right]+E_{x}[h(x, u)]
\end{gathered}
$$

By equation (74)

$$
\begin{gathered}
E_{x}[h(x, u)]=h(x)+E_{x} \int_{0}^{\alpha} A^{u^{*}} h^{*}(x) d t \\
h^{*}(x) \leq h(x, u)=
\end{gathered}
$$

$$
\begin{gathered}
E_{x}\left[\int_{0}^{\alpha} x^{T}(t) C(t) x(t)+u^{T}(t) G(t) u(t) d s\right]+h(x) \\
+E_{x} \int_{0}^{\alpha} A^{u^{*}} h^{*}(x) d t
\end{gathered}
$$

Or $0 \leq \mathrm{E}_{\mathrm{x}}\left[\int_{0}^{\alpha} \mathrm{x}^{\mathrm{T}}(\mathrm{t}) \mathrm{C}(\mathrm{t}) \mathrm{x}(\mathrm{t})+\mathrm{u}^{\mathrm{T}}(\mathrm{t}) \mathrm{G}(\mathrm{t}) \mathrm{u}(\mathrm{t}) \mathrm{ds}\right]$ $+\mathrm{E}_{\mathrm{x}} \int_{0}^{\alpha} \mathrm{A}^{\mathrm{u}^{*}} \mathrm{~h}^{*}(\mathrm{x}) \mathrm{dt}$

At $\alpha \rightarrow 0$. Thus $0 \leq \mathrm{E}_{\mathrm{x}}\left\{\mathrm{x}^{\mathrm{T}}(\mathrm{t}) \mathrm{C}(\mathrm{t}) \mathrm{x}(\mathrm{t})+\mathrm{u}^{*^{\mathrm{T}}}\right.$ (t) $\mathrm{G}(\mathrm{t}) \mathrm{u}^{*}$ (t) $\left.+\mathrm{A}^{\mathrm{u}^{*}} \mathrm{~h}^{*}(\mathrm{x})\right\}$

by equation (7) we have

$$
0 \leq x^{T}(t) C(t) x(t)+u^{* T}(t) G(t) u^{*}(t)+A^{u^{*}} h^{*}(x)
$$

Theorem (6). (convers of the HJB_equation)

let $\mathrm{h}^{*}$ (x) be a bounded function in $C(G)^{2} \cap \mathrm{C}(\mathrm{CL}(\mathrm{G}))$, Suppose that for all $\mathrm{u} \in \mathrm{Y}$ where $\mathrm{Y}$ is the set of control the inequality

$$
x^{T}(t) C(t) x(t)+u^{T}(t) G(t) u(t)+A^{u} h^{*}(x) \geq 0
$$

Then $h^{*}(x) \leq h(x, u)$, for all $u \in Y$, moreover $x^{T}(t) C(t) x(t)+u^{* T}(t) G(t) u^{*}(t)+A^{u^{*}} h^{*}(x)=0$, Then $\mathrm{u}^{*}$ is an optimal controle

Proof

Let $\mathrm{u}$ be a Markov control, and let u bea Markova control then

$A^{u} h^{*}(x) \geq-x^{T}(t) C(t) x(t)+u^{T}(t) G(t) u(t)$ for $u \in Y$

By equation (74)

$$
\begin{gathered}
\left.E_{x}\left[h^{*}(x)\right)\right]=h(x)+E_{x} \int_{0}^{T} A^{u} h^{*}(x) d t \\
\geq h(x)-E_{x} \int_{0}^{T} x^{T}(t) C(t) x(t)+u^{*^{T}}(t) G(t) u^{*}(t) d t
\end{gathered}
$$

Thus

$$
\begin{gathered}
h(x) \leq E_{x}\left[h^{*}(x)+\int_{0}^{T} x^{T}(t) C(t) x(t)+\right. \\
\left.u^{* T}(t) G(t) u^{*}(t) d t\right]=h(x, u)
\end{gathered}
$$

therefore

$\mathrm{u}^{*}$ is an optimal controle.

\section{Application 3 [Economics Model with Fractional Stratonovich Differential Equation]}

In 1928 F. R Ramsy introduced an economics model describing the rate of change of capital $\mathrm{K}$ and labor $\mathrm{L}$ in a market by a system of ordinary differential equation with $\mathrm{P}$ and $\mathrm{C}$ being the production and consumption rates respectively the model is given by

$$
\frac{d k(t)}{d t}=p(t)-C(t), \frac{d L(t)}{d t}=a(t) L(t)
$$

Where $\mathrm{a}(\mathrm{t})$ is the rate of growth Labor.

The production, capital and labor are related by the Cobb-Douglas formula.

$$
\mathrm{p}(\mathrm{t})=\mathrm{Ak}(\mathrm{t}){ }^{\alpha} \mathrm{L}(\mathrm{t})^{\beta}
$$


where A, $\alpha, \beta$ are some positive constant.

in certain the dependence of $\mathrm{P}$ on $\mathrm{K}$ and $\mathrm{L}$ is linear these mean $\alpha=\beta=1$ which will be our assumption throughout this section we shall also assume that the labor is constant, $\mathrm{L}(\mathrm{t})=$ $\mathrm{L}_{0}$; which is true for certain markets or relatively short time intervals of several years.

Therefore the production rate and the capital are related by

$$
p(t)=H(t) k(t),[1]
$$

A nether important assumption we make is that the production rate is subject to small random disturbances i.ep $(t)=H(t) k(t)+b(k(t)) \circ d B(t)$. therefore

$$
\frac{d k(t)}{d t}=H(t) k(t)+b(k(t)) \circ d^{H}(t)-C(t)
$$

Where $\mathrm{M}(\mathrm{t})=-\mathrm{C}(\mathrm{t})$

Which can be rewritten in the differential form as: -

$$
d k(t)=[H(t) k(t)+M(t)] d t+b(k(t)) \circ d B^{H}(t)
$$

Where $\mathrm{B}^{\mathrm{H}}(\mathrm{t})$ is Fractional Brownian motion $\mathrm{b}(\mathrm{k}(\mathrm{t}))$ is real function, characteristic of the noise, Assume that $\mathrm{M}(\mathrm{t})$ can be controlled the equation (84) become

$$
d k(t)=[H(t) k(t)+M(t) u(k(t))] d t+b(k(t)) \circ d B^{H}(t)
$$

usually one wants to minimize the cost function (75) let $\mathrm{g}$ ( $\mathrm{x}$ (T)) $=x^{T}(T) R x(T)$, and let $h^{*}(x) \in D\left(A^{\mathrm{u}}\right)$ and from definition (15) then (16) become

$$
\begin{gathered}
\left(A^{u} h^{*}\right) x=2 R x(T) H(t) x(t)+2 R x(T) M(t) u(t)+\int_{0}^{p} 2 R b \\
\text { (q) } b(p) \emptyset_{H}(p-q) d q
\end{gathered}
$$

Then (81) become

$$
\mathrm{h}^{*}(\mathrm{x})+\left(\mathrm{A}^{\mathrm{u}^{*}} \mathrm{~h}^{*}\right)=0
$$

$x^{T}(t) C(t) x(t)+u^{T}(t) G(t) u(t)+2 R x(T) H(t) x(t)+2 R x$

$$
\text { (T) } M(t) u(t)+\int_{0}^{p} 2 R b(q) b(p) \emptyset_{H}(p-q) d q=0
$$

by taking the derivative of two sides, one can get,

$$
\frac{d}{d u}\left[x^{T}(t) C(t) x(t)+u^{T}(t) G(t) u(t)+2 R x(T) H(t) x(t)\right.
$$

$$
\begin{gathered}
\left.+2 R x(T) M(t) u(t)+\int_{0}^{p} 2 R b(q) b(p) \emptyset_{H}(p-q) d q\right]=0 \\
2 G(t) u(t)+2 R x(T) M(t)=0 \\
u(t)=-\frac{\operatorname{Rx}(T) M(t)}{G(t)}
\end{gathered}
$$

Is optimal control for the linear-quadratic fractional Brownian motion differential equation and the optimal cost function is

$$
\begin{gathered}
h(x, u)=E\left(x(T)^{T} R x(T)+\int_{0}^{T}\left(x^{T}(t) C(t) x(t)+\right.\right. \\
\left.\left.\frac{(\operatorname{Rx}(T) M(t))^{2}}{G(t)}\right) d t\right)
\end{gathered}
$$

\section{References}

[1] A. F. Ivanov and A. V. Swishchuk, optimal control of stochastic differential delay equation. prepriat, December 2003, $6 \mathrm{pp}$ (Applied Math. Letters, sub-mitted).

[2] B. $\varnothing$ ksendal, Stochastic Differential Equations. May 2000, Springer-verlag Berlin Heidelberg New York.

[3] E. Allen, Modeling with ITO Stochastic Differential Equation. 2007-springer.

[4] F. P. Ramsey, "Amathemutical theory of savings". Economic J. 388 (1928), 543-549.

[5] Ganig., Heyde C. C., Jagers p. and Kurtz T. G., "probability and its Application", Springer-verlag London Limited, 2008.

[6] G. Gandolfo, "Economic Dynamics", springer-verlag, 1996.

[7] Javier R. Movellan, "Tutorial On Stochastic Differential Equation", 2011.

[8] K. E. Peter, Numerical solution of Stochastic Differential Equation. 1990 (springer-verlag Berlin.

[9] Nualart D., "Fractional Brounian Motion: stochastic calculus and Applications", proceeding Mathemati

[10] T. E. Duncan and B. pasik-Duncan, An approach to stochastic Integration for Fractional Brownian Motion in a Hilbert space. 